\title{
Les tragédies de l'assassinat et l'Hector d'Antoine de Montchrestien
}

\author{
ANTOINE SOARE
}

Formée, à tant d'autres égards, à l'école de Sénèque, la tragédie française tient aussi de son maître latin les rudiments d'une réflexion sur la problématique de l'héroïsme actif. Du temps où elle s'attardait sur le sort des vaincus, ne sachant dire encore de l'homme que l'être prévenu par la défaite, c'est en s'inspirant d'Hercules Oetaeus que Jacques de la Taille jetait ur. premier coup d'oeil, avec son Alexandre, sur le tragique propre à l'aventure des vainqueurs. ${ }^{1}$ L'échec dont il rendait compte ne se situait plus, comme si souvent dans la tragédie renaissante, en deçà de l'entreprise héroïque, devant quelque fatalité posée de l'extérieur et immédiatement inéluctable, mais sur les limites de l'efficacité, devant l'impossible déduit de l'immanence.

Les pièces qui relèvent, directement ou par l'intermédiaire de Jacques de la Taille, du modèle sénéquien s'échelonnent jusqu'au début de l'ère cornélienne. Hardy écrit lui aussi un Alexandre, tandis que Prévost donne une première version française d'Hercules Oetaeus, suivie des adaptations de Mainfray et de Rotrou. ${ }^{2}$ A l'écart de cette stricte filiation des sujets, la "figure tragique" qui leur est commune se retrouve dans La Mort d'Achille de Hardy, que reprendra Benserade, et aussi, sous une forme plus originale et plus complexe, dans l'Hector de Montchrestien. ${ }^{3}$

* * *

Le terme, tantôt avancé, de "figure tragique" requiert une définition, et le corpus que nous proposons, d'apparence tellement hétéroclite, quelques précisions méthodologiques. Si l'on admet que la tragédie a représenté, pendant plus d'un siècle de littérature française, une modalité privilégiée de la pensée sur l'homme, on ne saurait, à notre avis, en retracer correctement l'histoire sans recourir à une unité primaire de signification tragique, qui nous permette de repérer les permanences et les tournants de cette pensée. La nature des valeurs qu'une tragédie met en jeu ainsi que la nature et les rapports des forces dont elle fait dépendre ce jeu nous paraissent essentiellement et suffisamment constitutives d'une telle unité. Nous appelons "figures tragiques" les structures conflictuelles distinctes que l'on peut obtenir en variant ces données. Il va sans dire qu'une histoire de la tragédie qui procéderait par 


\section{4 / Renaissance and Reformation}

identification de ces figures aurait à pratiquer des découpages souvent différents de ceux auxquels nous ont habitués les critères de la technique dramatique, ou, du côté du contenu, les critères du "sujet" et du "thème." De ces deux derniers, surtout, on ne saurait assez redouter le pouvoir de brouillage, lorsqu'il s'agit de saisir, à travers les oeuvres, les mouvements de la pensée tragique.

Si par sujet l'on entend une séquence déterminée d'événements concernant un personnage historique ou légendaire précis, ce n'est pas encore là une unité pertinente de signification tragique, mais un exemplum seulement, qui peut, pour prestigieux qu'il soit, être assimilé à d'autres, par synonymie, ou différer de lui-même, par polysémie. Plusieurs sujets, puisés aux sources les plus diverses, peuvent ainsi être traités en référence à une seule figure tragique, celle-ci se présentant alors comme un modèle interprétatif applicable à une multitude de faits bruts fournis par la légende ou par l'histoire. De cette méthode de création dramatique, qui consiste à aborder l'univers des sujets avec l'idée préétablie d'une figure tragique, et que l'on pratiquera surtout durant la première moitié du siècle suivant, la reprise, par endroits littérale, d'Hercules Oetaeus dans la tragédie que Jacques de la Taille écrit sur Alexandre le Grand est un exemple patent et inaugural. Le fait que, dans son étude, par ailleurs tellement utile, sur L' "Hercule sur l'Oeta" de Sénèque et les dramaturges français de l'époque de Louis XIII, Jacques Morel ne mentionne aucune des pièces issues de ce dédoublement montre, à notre avis, combien lacunaires risquent d'être les corpus établis selon le seul critère du sujet. ${ }^{4}$

Inversement, aucune continuité du sens n'est à présupposer entre des pièces qui traitent le même sujet. Sous des éclairages dramatiques ou rhétoriques différents, celui-ci peut changer de relief sémantique au point qu'il se détache de la figure tragique qu'il illustrait auparavant, pour renvoyer à une autre, ou pour cesser tout simplement de signifier à la hauteur de la tragédie. La version, si curieusement composite, que Rotrou donne d'Hercules Oetaeus constitue, à cet égard, un cas limite, et nous ne l'avons incluse dans notre corpus que sous réserve d'en souligner la position marginale. Tout en reproduisant fidèlement la tragédie du héros de Sénèque, Rotrou la subordonne tellement à un épisode de son cru - une tragi-comédie du type Chryséide et Arimand qu'il la transforme en simple ornement mythologique de celui-ci. Devenues homologues, l'une de l'égarement, et l'autre de la conversion du tyran amoureux, l'agonie et l'apothéose du demi-dieu parviennent à peine à signifier encore par elles-mêmes. Le trajet eschatologique ne sert plus, en fin de compte, qu'à confirmer et à magnifier un trajet psychologique. Rotrou nous renseigne ainsi sur l'effacement plutôt que sur la persistance de la figure tragique que nous étudions, à l'époque, précisément, où celle-ci allait être définitivement abandonnée. ${ }^{5}$

Il arrive, enfin, qu'un même sujet renvoie à plusieurs figures tragiques à la fois, dans la mesure où les protagonistes peuvent, tout en formant un groupe 
dramatiquement cohérent, appartenir à des structures conflictuelles indépendantes les unes des autres. En recourant au philtre de Nessus, Déjanire déclenche, par exemple, deux tragédies, la sienne et celle de son époux, qui se posent en termes totalement différents. Jacques de la Taille ne retenait du modèle sénéquien, pour l'appliquer à son propre sujet, que la figure tragique centrée sur le personnage d'Hercule. Signe de l'intérêt que l'époque pouvait attacher à un certain aspect de la pièce de Sénèque, ce découpage est aussi un exemple précoce de la manière dont tendra à s'organiser, dans la tragédie française, l'univers des sujets, par cycles d'idées plutôt que d'événements.

Ceci dit, il reste que le sujet a plus qu'une fonction passivement exemplificatrice. Il soumet la figure tragique qu'il actualise à l'épreuve du concret dramatique, et constitue, de ce fait, un précieux moyen d'investigation. A ce titre, il mérite, sans doute, toute notre attention. C'est en se vérifiant à travers les multiples exemples auxquels la confrontent la légende et l'histoire qu'une certaine idée de l'homme, des forces et des valeurs qui le déterminent, vient à maturité.

Quant aux thèmes, avec tout ce que leur identification comporte d'arbitraire, ce ne sont, au mieux, que des sous-unités de signification tragique, sortes d'éléments morphologiques incapables de révéler d'eux-mêmes, et aussi longtemps qu'on les interroge séparément, la syntaxe qui les requiert. La critique thématique a ce défaut, du moins dans le domaine qui nous intéresse, de se contenter trop facilement de relevés paradigmatiques, avec le présupposé d'une facilité semblable dans le processus de création qu'elle étudie. Elle tend à réduire la filiation des oeuvres à une circulation de bribes et de fragments de toute nature et de toutes dimensions, pêchés et repêchés au hasard de leur dérive dans le courant de la tradition dramatique. Si elle permet de dépasser les cloisonnements qu'impose le critère du sujet, elle n'offre elle-même, le plus souvent, qu'une optique désarticulante.

On peut juger des avantages et de l'insuffisance du critère thématique par l'étude où Carla Federici met l'Alexandre de Hardy en rapport avec Hercules Oetaeus. ${ }^{6}$ En signalant une série de thèmes communs aux deux pièces, l'auteur prouve bien que l'une s'est inspirée de l'autre, malgré la différence des sujets, mais ne nous renseigne point sur la signification tragique qui ordonne ces thèmes dans le modèle, et que l'imitation reprend à son compte. Pour fructueuses qu'elles soient, de telles enquêtes ne laissent pas moins l'impression que les auteurs de tragédies n'auraient pratiqué l'art de redire que dans le décousu anthologique, avec, pour corollaire, exemption de repenser. Nous croyons, au contraire, qu'imiter, adapter ou transposer signifiait surtout, pour ces auteurs, réexaminer un itinéraire intellectuel complet, restituer, et approfondir, si possible, un sens intégral. 
Plus complexe que les thèmes qu'elle régit, et d'un ordre plus général que celui des sujets, dans la variété concrète desquels elle se répète, la figure tragique nous paraît donc définir le plan de signification sur lequel devrait se concentrer une histoire de la tragédie vraiment soucieuse de l'évolution idéelle du genre. D'une telle histoire, encore à écrire, nous n'esquisserons ici qu'un chapitre, modeste à en juger par les dimensions de notre corpus, mais aussi particulièrement éclairant, nous semble-t-il, quant au développement ultérieur du théâtre héroïque.

"Tragédies de l'assassinat," comme il conviendrait de les appeler, les pièces qui forment ce corpus associent à un même statut héroïque un même type de catastrophe. Leurs héros sont tous des guerriers parvenus au stade mythique de l'invincibilité, et dont la condition mortelle cesse, de ce fait, de constituer une évidence ternie par la résignation, pour apparaître comme un scandale moral et ontologique. Les invincibles ne peuvent mourir qu'assassinés, c'est-à-dire d'une mort nécessairement contraire à l'éthique qui informe leur vie, anéantis sur un plan de réalité inévitablement autre que celui sur lequel ils affirment leur être. La figure tragique qu'Hercules Oetaeus léguait aux dramaturges français est entière dans cette inadéquation. Sénèque en rendait compte au fil des tirades qu'il faisait prononcer au héros dévoré par la tunique teinte du sang de Nessus. Ses adaptateurs ainsi que les auteurs des deux Alexandre écriront des variantes de ce que l'on pourrait appeler, d'un terme générique, "le discours de l'invincible agonisant." Dans Hector et dans les deux Achille, où les héros n'auront guère le temps de dire leur mort, une variété de moyens dramatiques seront utilisés, au lieu du développement rhétorique unitaire, pour signifier la même figure tragique. Nous analyserons d'abord celle-ci, telle, seulement, qu'elle apparaît dans son expression discursive. Il nous sera ensuite plus aisé de la reconnaître dans le contexte élargi et diversifié où la situe la pièce de Montchrestien.

Le discours de l'invincible agonisant suppose une situation pathétiquement invraisemblable, factice par fidélité à la logique contrariée du surhumain: les héros se meurent sans pouvoir comprendre ce qui leur arrive. Ils s'interrogent, ces héros, sur le mal qui s'est emparé de leur corps, avec une stupéfaction, et lui reprochent sa félonie avec une candeur que l'on dirait antérieures au savoir le plus élémentaire de l'espèce. Leur parole est moins gémissement de la chair souffrante ou plainte de l'âme délogée que protestation de l'esprit devant l'inintelligible:

Sors sors quiconques fais dans moy ta garnison,

Vien avant, sans me prendre en telle trahison.

Pourquoy assailles tu mes membres en cachette?

Di moy qui t'a donné l'entree si secrette,

$O$ peste, dans mon corps? Deà quel rivage more?

Quelle terre Pontique, et quelle Circe encore

T'a produit pour domter Alexandre invincible? 
Es tu quelque couleuvre, ou quelque aspic horrible?

Lás dy moy qui tu es: qu'à tout le moins je sçache

Qui est mon ennemi, qui dedans moy se cache,

Pour me faire mourir: certes quiconques sois,

Main à main contre moy venir tu n'oserois.

(la Taille, Alexandre, v. 865-876) ${ }^{7}$

Ignorance aussi radicale des voies ordinaires de la mort qu'est exclusive la foi trahie dont elle procède. Des effets hyperboliques désignent et garantissent ici le superlatif postulé. La tragédie des invincibles est en proportion de leur adhésion, dont il nous faut précisément admettre le caractère absolu, au modèle existentiel de la lice, le seul selon lequel mourir leur est, par conséquent, concevable, et par définition impossible. Moins étranger aux choses de l'homme que ses homologues, l'Alexandre de Hardy remarque lui-même l'extravagance des propos que lui dictent ses réflexes de conquérant. $\mathrm{La}$ naïveté de l'agonisant, ailleurs acceptée sans commentaires, mesure explicitement chez lui l'incompatibilité entre la représentation guerrière et le fait commun de la mort:

Inuisible ennemy, serpent à qui l'enuie

Sur le caucase horrible en froideurs donna vie,

Traître pren quelque forme, \& sorti de mon sein,

Sans me pouuoir venger, ne m'étouffe, assassin,

Fay qu'un iuste combat vuide nôtre querelle,

Ma gloire t'absoudra si tu gaignes sur elle,

Eusses-tu mis en vn les cors de Gerion,

Les forces d'vn Antée, \& celles d'Orion:

O Cartel insensé, ridicule menace

Vers l'extrême douleur que les membres me glace ...

(v. 893-902)

Pour le guerrier, dont les Hercules et les Alexandres réalisent tragiquement l'idéal, la mort ne saurait être qu'un des termes glorieux de l'alternative que fait surgir le combat singulier. On ne meurt, en lice, qu'à défaut de vaincre, c'est-à-dire par et pour seulement le plus haut que soi, en consacrant sa vertu, au-dessus de tous les triomphes qui pourraient l'illustrer, par la vertu plus grande de l'adversaire. Associé à la transcendance de son vainqueur, chaque guerrier acquiert en mourant plus de gloire qu'il ne saurait obtenir à l'intérieur de sa vie. A la fois productrice du seul bien héroïque et convertible en lui, cette vie est faite pour cesser à l'instant, forcément magnifiant, où sa valeur d'échange excède sa valeur d'usage.

L'alternative du "vaincre ou mourir" régit ainsi un système de troc héroiqque, où l'anéantissement de l'être physique a pour contrepartie obligatoire un suprême exhaussement de l'être moral. En ramenant, d'autre part, la mort à une affaire de vertu à vertu, elle promet à l'individualité guerrière un statut ontologique d'exception, sans rapport avec la malédiction qui pèse indistinctement sur le reste de l'espèce. Mourir au combat, de cette mort dont on assure 
par l'épée les conditions et le sens, c'est justement tourner au profit de la différenciation héroïque le phénomène par lequel se manifeste ailleurs le plus péniblement l'uniformité de l'humain. A l'ordre de la mortalité naturelle, la lice superpose et oppose, en somme, son propre ordre de mortalité, où la dépense en pure perte des ressources vitales est corrigée par une économie du sublime, et la promiscuité du destin collectif par une hiérarchie des courages. Le guerrier fait voeu de n'exister que dans et par cet ordre second, auquel il s'efforce de conférer étanchéité et permanence en affrontant sans cesse une mort sans cesse incarnée dans des adversaires de plus en plus valeureux.

A tous égards, l'assassinat intervient comme une revanche de la condition commune sur l'élitisme guerrier. En s'attaquant au corps sans solliciter la vertu, il enlève la vie sans fournir son équivalent en gloire. Il constitue, en termes d'économie morale, la fraude par laquelle arrivent les faillites dans le système du troc héroïque qu'avait institué la lice, et s'opère le retour au système du "tribut" payable à la nature. Si les invincibles agonisants demandent raison au tribunal des dieux de leur mort sans combat comme d'une affreuse aberration, c'est que par elle ils se font littéralement voler leur vie, qu'on les empêche de jamais acquérir le "titulus extremus" - "le tiltre de leur tombeau," comme traduisait Prévost ${ }^{8}$ - en les dépouillant du seul avoir qu'ils pouvaient offrir en échange:

Fallait-il que vaincu je fusse sans vainqueur?

Si je mourois au moins par quelqu'un dont l'honneur

Mesme honorast ma mort: mais je vais en enfer

(O les lasches destins) sans mettre en main le fer.

(la Taille, Alexandre, v. 899-902)

Ontologiquement parlant, d'autre part, l'assassinat ramène ses victimes à l'état ignominieux que leur assignait d'emblée la nature, celui d'objets impuissants et indifférenciés d'une agression anonyme. Il replonge l'individualité guerrière dans la servitude qui afflige le troupeau, et dont il n'est, en fait, lui-même qu'une actualisation précipitée. Périr autrement que par l'effort loyal des armes revient justement, quelles que soient les circonstances particulières de la fin, à périr comme un chacun, par la seule misère du corps. Si le thème du "trépas vergogneux" figure dans toutes les tragédies que nous étudions, dans l'Alexandre de Jacques de la Taille, par exemple:

Ay-je doncques la mort si honneste eschappee

Par plus de mille fois, àfin de l'avoir telle

Que quelque homme couhart ou craintive pucelle? (v. 912-914)

ou dans l'Hercule de Prévost:

Perdi-ie tant de fois une mort honorable

Pour perdre mon beau tiltre et mourir miserable ... (acte IV, p. 27) 
ou, enfin, dans l'Alexandre de Hardy:

Combien là du trépas me plaisoit la fortune,

Non dans vn lit pareil à l'ignoble commune,

Peu à peu deuoré par ce tourment maudit ... (v. 1125-1127)

c'est que ce thème porte sur un aspect essentiel d'une figure tragique commune: la dégradation ontologique que l'assassinat inflige aux invincibles, en les faisant choir de l'ordre de la mortalité guerrière dans l'ordre de la mortalité naturelle.

A cet égard, la tunique que Déjanire fait revêtir à son époux ou le poison qu'Antipatre fait boire à son roi sont, d'ailleurs, par leurs effets, d'un symbolisme transparent. Qu'est-ce, à proprement parler, que cet embrasement de la chair que si recroqueville et qui fond à vue d'oeil, ou ce feu qui consume les entrailles avec une implacable promptitude, sinon le métabolisme ordinaire de la vieillesse et de la mort, reproduit en accéléré? ${ }^{9}$ L'effet, purement dramatique, de vitesse mis à part, c'est un même ravage que celui des venins magiques et des ans; et c'est avec l'effarement de l'extrême sénescence que les jeunes agonisants mesurent la ruine de leur corps:

Est-ce doncq là ce bras qui nettoya la terre

Des monstres, des tyrans qui luy faisoient la guerre?

...

Le Ciel s'est-il assis sur ces espaules là?

...-

Ce pied d'vn pas soudain

A-t-il onc emporté de vitesse le daim?

(Prévost, Hercule, acte IV, p.23)

$\mathrm{O}$ proüesse, ô vigueur de iadis, où es-tu?

L'enuieuse Cloton te dérobe, voisine,

Et ma parole meurt, recluse en la poitrine.

(Hardy, Alexandre, v. 964-966)

Derrière les efforts et le décor de l'héroïsme guerrier, l'assassin révèle la permanence de l'humain. Il réduit ses victimes à une condition qu'elles n'ont jamais vraiment quittée, et c'est pourquoi sa tâche est d'une facilité aussi prévisible que stupéfiante. Emissaire et restaurateur de l'ordre naturel, il met fin par aplatissement à une ambiguiité d'être qu'aucun arrachement n'est suffisamment radical pour trancher en faveur d'un ordre supérieur, et que la mort guerrière même ne peut qu'escamoter. Les tragédies de l'assassinat prennent, le plus souvent, pour prétexte dramatique une manifestation morale quelconque de cette ambiguïté, leurs intrigues évoluant ainsi du symptomatique vers le patent. Les défauts par lesquels les héros trahissent leur appartenance à l'espèce - désordre passionnel ou abus du pouvoir - sont ceux aussi qui occasionnent leur fin vulgaire. La formule est séduisante par son équilibre 
aristotélicien, mais n'induit que trop facilement à abaisser le tonus tragique au niveau de la simple moralisation. Pour qu'un tel appauvrissement du sens se produise, il suffit que la série d'oppositions homologues qui préfigurent le dénouement soit présentée comme une série causale d'événements qui le justifient. C'est ce qui se passe dans l'Achille de Hardy, où l'égarement amoureux du héros, au lieu d'annoncer, par conformité structurale, l'outrage final - comme c'est encore le cas dans Hercules Oetaeus et dans les pièces qui s'en inspirent directement - le provoque tout simplement, comme le péché sa peine. Inversement, un héroïsme exempt de toute faiblesse, tel que l'imaginera l'auteur d'Hector ne rendra que plus proprement tragique son impuissance à transcender la condition des mortels.

Les invincibles se meurent donc, spoliés et humiliés, du mal de l'homme. Alors que leur désespoir est de reconnaître, dans l'assassinat dont ils sont fortuitement les victimes, des ressemblances honteuses avec la mort naturelle, ils n'ont, précisément, affaire qu'à celle-ci, pour eux inévitable, et à son tour perçue comme un assassinat. C'est parce que les deux termes se signifient réciproquement, et qu'il y a ainsi va-et-vient du sens entre le contingence de l'un et la fatalité de l'autre, que l'aventure des invincibles se présente non pas tout simplement comme un fait divers particulièrement dramatique, mais comme un théorème tragique. La lice ne parvient, en effet, à maintenir un ordre privilégié de mortalité qu'en prévenant la mort naturelle par la mort guerrière, mais elle reporte, d'autre part, celle-ci au bout d'une série de victoires que seule peut interrompre la victoire d'un adversaire plus valeureux. Alors qu'elle fonde ainsi son ordre sur la relativité de la valeur guerrière sujette à une continuelle surenchère, elle oriente, par là même, l'effort héroïque vers un absolu qui échappe à sa juridiction, et que récupère forcément l'ordre de la mortalité naturelle. Abandonnés de tous leurs adversaires sur le sommet prodigieux que tout guerrier mourait de ne pouvoir atteindre, contraints au repos faute de combattants, ${ }^{10}$ les invincibles se retrouvent voués, comme au plus bas de l'échelle humaine, aux affres de la mort-assassinat. Leur misère est comprise dans l'excès de leur triomphe, paradoxe dont ils diront l'ironie en évoquant leurs exploits de jadis comme autant d'occasions manquées, en rêvant de l'impossible défaite qui les aurait diminués pour sauvegarder leur gloire:

Ay-je donc eschappé tant de playes et de coups

Qu'on m'a fait en la guerre? Ay-je donc le courrous

Vaillament evité du mutin Ocean?

Me suis-je preservé des couleuvres Indoises,

Ay-je lás enduré tant de maux et malaises,

Ay-je sain traversé des desserts plus de mille

Pour si honteusement mourir en ceste ville?

(la Taille, Alexandre, v. 1004-1010) 
Les tragédies de l'assassinat concluent, en somme, à l'absurdité de l'idéal guerrier. Elles le font suivant un mouvement qui n'est pas sans rappeler celui de l'enquête oedipienne, en repliant sur l'identité des protagonistes l'altérité dont ils s'obstinent à poursuivre les manifestations extérieures. Cette mort qu'il ne conçoit qu'incarnée dans un adversaire, le héros guerrier la porte en lui-même, comme le héros justicier le crime. Un Hercules furens fait justement pendant, chez Sénèque, à Hercules Oetaeus, et ce qu'il faudrait surtout remarquer dans ce diptyque n'est pas tant l'unité biographique des sujets que la complémentarité des figures tragiques. Une déception en deux temps sanctionne la double illusion du triomphe herculéen. L'exterminateur des monstres et le vainqueur des enfers découvre l'un après l'autre, en lui-même, les fléaux dont il croyait avoir purgé la terre: le crime et la mort. "Vnde hic cruor?," s'écriait-il en revenant de ses fureurs, "Vbi morbus, ubinam est?", s'écrie-t-il dans son agonie. ${ }^{11}$ Questions analogues, présidant, l'une en registre moral, et l'autre en registre ontologique, à la résolution des manichéismes mythiques en ambivalences de tragédie.

Théorème tragique, disions-nous, alors que l'échec des invincibles semble tenir plutôt du truisme. C'est que les tragédies de l'assassinat situent justement l'évidence la plus flagrante dans une perspective de malentendu. Telle est la démarche qu'elles adoptent pour dire, en fin de compte, ce qu'il y a d'irréductiblement scandaleux dans le déclin et dans la mort de l'homme. L'alternative du vaincre ou mourir leur fournit de quoi légitimer une protestation que découragerait autrement la banalité d'un savoir faussement résigné. Dans son exclusivisme, elle fait passer pour naturalisé l'ordre de mortalité que la lice n'oppose que préventivement et par convention héroïque à l'ordre naturel. Le guerrier fonde son espoir le plus follement ambitieux sur cette substitution du droit au fait, dont l'impossibilité appelle dès lors le supplice de la démonstration. Aveuglé par sa loyauté, il est aussi incapable de concevoir la perfidie de la mort naturelle que prompt à assimiler l'invincibilité à l'immortalité. Si la mort ne doit arriver que par l'adversaire, celui qui triomphe de tous ses adversaires doit triompher de la mort même: tel est le raisonnement qui se loge, discret, mais tenace, jamais pleinement formulé, ni totalement découragé, au plus profond de son esprit.

L'éthique du combat singulier cautionne ainsi, sans pouvoir le soutenir, un projet de salut par l'effort des armes, précurseur, et équivalent en registre paien, du projet chrétien de salut par l'effort de la foi. Les invincibles sont victimes d'un mirage eschatologique, et jugent de la condition mortelle en rédempteurs déçus. ${ }^{12} \mathrm{Il}$ n'est nullement évident, à leurs yeux, mais incompréhensible, et combien révoltant, qu'ils doivent rejoindre l'homme au terme d'une carrière dont chaque étape semblait les rapprocher un peu plus de la divinité. Parmi les auteurs français, Hardy a trouvé les paroles les plus émouvantes pour dire quelles sont, dans les tragédies de l'assassinat, les véritables proportions de l'enjeu et de l'échec: 


\section{2 / Renaissance and Reformation}

Absurde vanité, impieuse folie

Produite des vapeurs d'vne melancolie,

Qui dedans mon cerueau ne penetra iamais,

Ie ne crus onc amis, ne croiray desormais,

Autre qu'humain tirer l'origine mortelle,

Les Dieux n'ont avec nous aucune parentelle ...

(Alexandre, v. 941-946)

Alexandre se défend, sans doute, d'avoir nourri un espoir tenu pour sacrilège par son public chrétien, mais son discours ne se ressent pas moins de l'amertume auquel, dans sa sincérité païenne, l'Hercule de Sénèque donnait jadis libre cours. L'universalité de la mort est traitée d'ailleurs, dans les épilogues des tragédies de l'assassinat, comme un objet d'enseignement:

Puis esperez humains vn bon-heur de durée,

Vne grandeur qui soit icy bas asseurée,

Présumez que tenir le monde sous vos loix,

Que porter dans le Ciel vos belliqueux explois,

Obtienne de la parque vn moindre priuilége,

Que le triste Minos ne vous cite à son siege,

Erreur, énorme erreur, richesse ne beauté

Ne peuuent empêcher ce destin qui moissonne

Les hommes iournaliers \& n'excepte personne,

De la terre venus $\&$ en elle resous,

Même sort à la fin leur est égal à tous;

Epreuue trop cruelle, épreuue trop certaine,

Qui, ce Monarque étient, rend nôtre attente vaine ...

(Hardy, Alexandre, v. 1225-1238)

De telles réflexions, qui pourraient passer, dans un autre contexte, pour un lieu commun de la rhétorique humaniste, font effectivement figure de corollaire dans le contexte guerrier, où, à mi-chemin entre la résignation hébétée et la consolation chrétienne, l'invincibilité oppose à l'évidence des arguments dérisoirement légitimes.

Une dernière question se pose: les tragédies de l'assassinat ne sépareraientelles pas, pour mieux dresser leur constat d'échec, l'effort individuel de l'effort qui se perpétue à l'échelle collective de la race? La permanence du sang généreux n'offrirait-elle pas, autrement dit, de quoi rémédier à la précarité du corps? Exclue sans commentaires, une telle possibilité ne se dessine qu'en creux dans Hercules Oetaeus, où une délégation strictement amoureuse du père au fils souligne l'absence de la délégation héroïque. ${ }^{13}$ Sénèque s'en tenait à une solution par la verticale de l'individu: sa tragédie aboutissait, tant bien que mal, et après avoir déployé, d'ailleurs, un sens complet dans l'immanence, à une apothéose païenne. Ce dépassement dans la direction du sacré aura perdu son crédit à l'âge chrétien sans que les auteurs français se montrent, pour autant, plus curieux d'un dépassement dans la direction profane de la race. ${ }^{14}$ Plutôt que d'une omission conventionnelle, on 
pourrait dire qu'il s'agit là d'une impossibilité d'emblée reconnue et traitée comme telle. Sans égal dans le temps aussi bien que dans l'espace, l'invincible est précisément celui qui n'a pas de successeur, par excès de valeur exclu du milieu où se reproduit la valeur, comme du milieu où elle se fait consacrer. Aussi sa mort apparait-elle, dans la plupart des tragédies que nous étudions, comme une perte totale et définitive, échec sans revanche de l'humanité guerrière en son entier.

Hardy se distingue toutefois, à cet égard, de ses prédécesseurs. Peut-être inspiré déjà par une curiosité naissante pour l'être historique de l'héroïsme, et qui allait conduire, dans les décades suivantes, à un complet remaniement du théâtre tragique, il examine de près l'hypothèse du salut par la filiation. Ses tragédies de l'assassinat finissent sur une note non plus de désespoir, mais d'anxiété, dans l'entre-deux d'une relève hérö̈que mal assurée. Achille laisse en mourant un fils aux Grecs, mais qui pourrait trahir l'espoir qu'on met en lui, car il n'a pas encore fait ses preuves, et le sang n'est point, comme le rappelle Ulysse, le plus sûr garant de la valeur:

Maints ont degeneré, et toûjours ce compas,

Enuers les successeurs infaillible n'est pas..

(Achille, v. 1715-1716)

A cette incertitude quant à l'avenir moral de la lignée, s'ajoute, dans Alexandre, l'incertitude quant à son avenir physique. Le père meurt avant que naisse le fils:

Rosane enceinte peut vous laisser après moy

(Foible attente pourtant) quelque surjon de Roy.

(v. 1134-1135)

Rien de mieux pour faire ressortir l'insuffisance des moyens que ce flottement des effets. Quand même l'invincibilité pourrait survivre en autrui, cette survie demeurerait aléatoire, et donc temporaire, dépendante elle aussi de l'ordre non pas de la vertu, mais de la nature. La race ne transcende pas, elle élargit tout au plus, la tragédie qui se consomme, sur le plan de l'individu, par la mort-assassinat. Solitaire ou multiplié dans la durée généalogique, le corps installe, entre l'effort des armes et son ultime objectif, un obstacle infranchissable.

Telle est, précisément, la situation à partir de laquelle s'élaborera le programme eschatologique de l'héroïsme cornélien. Le Cid commence par une tragédie de l'assassinat, résumée en quelques scènes et dépouillée d'affabulations symboliques. Don Diègue est consumé par l'âge, comme les Hercules et les Alexandres l'étaient par quelque venin. Pour avoir survécu à toutes les échéances glorieuses de la lice, il subit les viles rigueurs de l'ordre, où il est retombé, de la mortalité naturelle. C'est la misère de cet état équivoque de guerrier vieilli, dissimulée tant bien que mal derrière la respectabilité du courtisan, que la querelle avec le Comte remet à nu. 
La tragédie que l'assassin enseignait, si l'on peut dire, en la produisant, le père de Chimène la dénonce tout simplement, en soldat insurgé contre les compromis de la vie civile. Il n'a plus besoin de provoquer lui-même la déchéance physique de son rival, il lui suffit de lui restituer de force sa signification primitive. Les raisons de blâmer sa conduite ne manquent certes pas, mais elles n'ont rien à faire avec le fond de la question. Du strict point de vue de la morale et de l'ontologie guerrières, l'aberration est du côté de Don Diègue. L'ordre de mortalité seconde qu'institue la lice exclut précisément le phénomène de la vieillesse. Le moindre décalage entre l'être de vertu et l'être de chair y est censé entraîner sur le champ une fin glorieuse. Le guerrier meurt dès qu'il cesse de vaincre, c'est-à-dire toujours à l'apogée de sa carrière, au point le plus haut de coïncidence entre ses forces et son courage. La vie sans déclin, tel est au fond le choix que l'élite armée oppose à l'ignominie de la mort naturelle. Instruit par l'affront qu'il vient de subir, Don Diègue définira lui-même la limite idéale, et par lui tragiquement dépassée, de la longévité guerrière:

\section{Qu'on est digne d'envie}

Lorsqu'en perdant la force on perd aussi la vie, Et qu'un long âge apprête aux hommes généreux, Au bout de leur carrière, un destin malheureux!

$$
(\text { v. } 697-700)^{14}
$$

Bien avant lui, les invincibles en instance d'assassinat ajoutaient à l'ironie de leur fin en affichant le mépris, caractéristique de leur caste, pour la vieillesse qu'ils faisaient voeu d'éviter:

$$
\text { ... fy, fy de ce vieil age }
$$

Qui nous dénue tous de force et de courage.

O Dieux ne permettez qu'une mort languissante

Me face aller chenu jusques à Rhadamante.

(la Taille, Alexandre, v. 445-448)

Le fameux monologue du vieillard:

O rage! ô désespoịi ô vieillesse ennemie!

N'ai-je donc tant vécu que pour cette infamie?

Et ne suis-je blanchi dans les travaux guerriers

Que pour voir en un jour flétrir tant de lauriers?

(v. 237-240)

et sa reprise, sous forme de plaidoyer, devant le roi ne sont, on peut s'en rendre compte maintenant, qu'une dernière version du discours de l'invincible agonisant. Sans que l'hypothèse mythique de l'invincibilité joue ici aucun rôle. Le malheur que clame Don Diègue ne résulte plus que des restrictions que la discipline civique impose à la quête farouchement individualiste du 
salut guerrier. La cité entend récupérer et revaloriser les ressources qu'elle ne peut plus employer, sans risques inutiles, à sa défense, et ses lois réhabilitent une condition méprisable à en juger par la seule loi du vaincre ou mourir. Héros en service commandé, Don Diègue a accepté de quitter les champs de bataille aux premiers signes du déclin, au moment même où il aurait pu troquer sa vie contre la gloire suprême des armes. S'il est parvenu maintenant à l'âge malheureux où il ne peut même plus engager un combat, c'est qu'il n'a pas livré alors le combat qui aurait dû être, pour lui, le dernier. Sa rechute dans l'ordre de la mortalité naturelle a pris la forme anodine d'une mise à la retraite. Tellement anodine, qu'il se considère encore en droit d'en parler, quelques instants avant l'affront, sans honte et sans amertume:

Quand l'âge dans mes nerfs a fait couler sa glace

Votre rare valeur a bien rempli ma place ...

(v. 209-210)

On trouve en lui un parfait exemple de la manière dont la routine sociale parvient à assoupir, par souci de l'avenir collectif et moyennant la vénération garantie aux exploits du passé, le désespoir métaphysique qui rive le guerrier au présent frénétiquement répété de l'épreuve héroïque. ${ }^{15} \mathrm{Ce}$ désespoir, le Comte le vit pleinement, sur le mode de l'orgueil, et le ranime malignement chez son rival, sur le mode de l'humiliation.

Le ressort de la filiation fonctionne cette fois vigoureusement. Rodrigue restaure l'intégrité, compromise en Don Diègue, de l'être guerrier. Mais s'il surpasse en valeur tous ses ancêtres et tous ses adversaires, ce n'est que pour rejoindre les hauteurs mythiques de l'invincibilité, la région par excellence des tragédies de l'assassinat. Corneille connaissait trop bien la tradition tragique dont il livrait l'essentiel au début de sa pièce pour ignorer les résonances fatidiques du titre qu'il conférait à son héros à la fin. ${ }^{16} \mathrm{~L}$ 'échec, tel que Don Diègue le vit et l'exprime, de l'eschatologie guerrière, Rodrigue ne fait que le reporter au-delà du splendide sursis de sa carrière, à l'extrême échéance où le situaient d'emblée les pièces d'inspiration sénéquienne.

Si une véritable solution s'ébauche toutefois, c'est que dans l'entre-deux de la relève, et par l'effort même qui rend celle-ci possible, la problématique de l'héroïsme actif est définitivement transportée sur un autre plan. On n'écrira plus, après Le Cid, des tragédies de l'assassinat. Par un consensus à l'échelle de toute une génération de dramaturges, l'héroïsme cessera de protester inutilement contre le fatum physique, pour investir son exigence de surhumanité dans les défis et dans les sacrifices de la transcendance morale. Il changera aussi, avec Corneille, de stratégie, pour progresser par une série d'hypostases distinctes vers l'objectif qui lui demeurait inaccessible au seul niveau de l'hypostase guerrière. L'histoire réelle de Rome, telle qu'elle apparaît à travers les trois âges d'Horace, d'Auguste et de Polyeucte, sert de modèle à une histoire idéale de l'homme, conçue comme une succession de fonctions 
héroïques dont chacune dispose de moyens plus efficaces et d'un champ d'action plus vaste que la précédente. Au terme d'une quête commencée par le guerrier et relancée par le souverain, le martyr réalise le voeu héroïque d'immortalité, une apothéose chrétienne répare, dans le ciel de Polyeucte, le scandale païen de la mort-assassinat.

* * * *

L'Hector de Montchrestien situe la problématique de l'héroïsme actif sur le plan traditionnel, familier à un Jacques de la Taille, à un Prévost ou à un Hardy, mais en fournit une analyse d'une ampleur et d'une netteté qui font de lui la tragédie la plus cornélienne avant la lettre. Véritable compendium des aspirations et des servitudes de l'être guerrier, il articule un réseau d'inadéquations secondes autour de l'inadéquation centrale qui se manifeste dans l'assassinat, il se présente, à proprement parler, comme une tragédie globale de l'incarnation. Alors que les Hercules et les Alexandres poursuivaient leur quête dans une sorte de vide social où ils ne rencontraient que l'obstacle solitaire de leur propre corps, le héros de Montchrestien est pris dans le tout de la pâte humaine, la chair qui l'embarrasse comme empêtrée à son tour dans la chair collective de la famille et de la cité. Aussi sera-t-il tout d'abord confronté à un conflit d'un ordre plus général, que résume le dialogue stichomythique avec Priam, entre la vocation du guerrier impatient de valoriser sa mort et les obligations du chef responsable de la survie du groupe, entre la gloire et la prudence.

Hector ne se laisse plus séduire, d'autre part, comme ses homologues de la tradition sénéquienne, auxquels il fait justement allusion, par le mirage eschatologique d'une immortalité physique à acquérir à force d'invincibilité:

Il nous faut tous finir, tu ne l'ignores pas,

Je ne suis engendré de semance immortelle;

Et si les fils des Dieux ont chargé la nasselle

Dont le crasseux Nocher trajecte l'Acheron

Se faut-il esbahir que nous autres mourons?

(v. 348-352)

Mais s'il ne compte plus échapper à sa condition mortelle, il n'entend aussi l'assumer que sur lé mode magnifiant de l'épreuve héroïque:

Aussi me faut-il vaincre ou mourir au combat. (v. 206)

Au partage stoïcien qu'il fait entre les libertés et les sujetions, le droit de regard sur la mort lui apparaît précisément comme l'apanage de la volonté guerrière:

Combattre et bien mourir dépend au moins de nous. (v. 698)

...

L'espoir d'un coeur vaillant ne dépend que de luy. (v. 790) 
Le dénouement de la pièce devra démontrer encore une fois, et telle sera sa véritable fonction tragique, la vanité de l'effort, ici déployé avec le plus de véhémence et de légitimité, pour fonder l'existence sur l'alternative du vaincre ou mourir.

Ce n'est qu'une immortalité morale qu'Hector espère encore acquérir, à force de gloire. Avec une horreur du corruptible et une soif d'éternité qui font de lui un digne pendant guerrier de Polyeucte, il cherche dans l'éclat de ses exploits un principe de salut. Mais la gloire risque de s'engluer dans la prudence du chef, comme la valeur s'englue dans le corps, et sans cesse exposée à des compromis, elle offre au jugement d'autrui la même prise facile et déloyale que la chair offre à l'assassin. L'une est à la merci de la médisance comme l'autre à la merci du coup félon. Le héros de Montchrestien en est conscient jusqu'à l'obsession. Sa tragédie commence dans l'angoisse, que l'Horace de Corneille éprouvera au dénouement de la sienne, de cette autre forme d'assassinat:

Mais je crains la vergogne à jamais reprochable,

Je crains les traits piquans d'un peuple variable,

Leger, presomptueux, sans respect et sans loy,

Qui desployant sa langue à blassoner de moy

Tournerait ma prudence en lasche coüardise.

Bien tost se perd la gloire à grand labeur acquise ...

Les tensions accumulées durant les trois premiers actes se relâchent brusquement au début du quatrième. Hector rompt les encerclements de la famille et de la cité, il se dégage de toutes les attaches sociales de l'incarnation, pour surgir dans cet espace idéal de liberté et de droiture que représentent pour lui les champs de bataille. Dans ce grand dehors de la gloire, dont il n'avait cessé de rêver derrière les remparts de Troie:

\footnotetext{
$\mathrm{O}$ trois fois bien-heureux sur tout autres j'estime

Qui dispose à son gré d'un dessein magnanime,

Sans être inquieté par les exhortements

D'un père apprehensif, par les embrassements

Que joint à ses baisers une femme agreable,

Par les voeux respectez d'une mère honorable,

Par les graves conseils des vieillards reverez,

Quant di-je à la vertu ses efforts sont tirez.
}

(v. 1045-1052)

Percée aussi impétueuse qu'illusoire: au dynamisme libérateur du geste correspond une disposition en entonnoir du sens. La tragédie reflue vers son centre, elle se ramasse dans son foyer originel, à la faveur même du mouvement qui paraissait la dissiper. Hector emporte avec lui le principe indéracinable, et maintenant ramené au premier plan, des servitudes qu'il croit avoir abolies, son propre corps, par la misère duquel il périra, comme tous les 
invincibles, sans combat et sans gloire. Il ne triomphera de Polybète, selon les normes loyales de la lice, que pour devenir aussitôt la victime d'Achille, dans l'ordre ignoble de la mortalité naturelle. Victoire et mort du héros se succéderont à l'intérieur d'une seule séquence dramatique, l'alternative guerrière sera bafouée par la juxtaposition:

Hector d'un oeil ravi mesure sa grandeur,

Fait branler son pennache en la claire splendeur

Du casque flamboyant qui gist dessus la terre,

Et veut s'orner le chef de cet astre de guerre.

Le corps estendu mort il taste à plusieurs fois

Pour voir s'il demouroit veuf d'esprit et de voix;

Puis le fait despoüiller par l'un de ses Gendarmes

Du fardeau glorieux des reluisantes armes.

Mais prest à se courber pour enlever l'armet,

Achille survenu derriere luy se met,

Ses mouvements espie, observe sa demarche;

Et voyant que son corps se courbait comme une arche

Penché dessus la terre, aux reins il l'enfonça,

De sorte que le fer jusqu'au coeur traversa.

Hector torne à l'instant et le frapper essaye;

Mais il sent eschapper son ame par sa playe.

(v. 2175-2190)

Le coup d'épée dans le dos n'a sans doute pas les mêmes vertus symboliques que le poison; il laisse moins paraître les similitudes qui font de l'assassinat un substitut de la mort naturelle. Mais l'art du récit supplée largement à l'insuffisance sémantique de l'événement. Nulle part, dans les tragédies de l'assassinat, le corps n'aura mieux exhibé la plastique de sa vulnérabilité, ni la chair étalé plus massivement son indolence que dans les vers où Montchrestien raconte la fin de son héros. L'invincible tourné tout entier ves sa gloire tandis que la mort se dessine derrière lui, dans la silhouette sournoise de l'assassin: la scène, on dirait un bas-relief funéraire, figure de la manière la plus saisissante l'irrémédiable dualité de l'être guerrier.

Hector abattu, Achille s'empare facilement de Troie, C'est le deuxième temps d'une même agression félonne. Le massacre fait suite à l'assassinat, l'un vérifiant sur l'ensemble de la collectivité héroïque la précarité que l'autre avait révélée dans le corps du héros. La tragédie regagne ainsi, par un dernier rebondissement, sa dimension sociale. Le choeur se lamente:

Par la dextre d'Hector Troye a resté debout;

Par sa mort malheureuse elle tombe du tout.

Il faut bien qu'elle soit de nos larmes suivie;

En elle nous perdons la victoire et la vie.

Que le bonheur public est foible et vacillant

S'il depend de la main d'un seul homme vaillant,

Qui s'offre à tous hazards sans crainte de la Parque.

(v. 2375-2381) 
Il ne pouvait en être autrement. L'idéal de l'invincibilité met inévitablement le groupe sous la dépendance d'une individualité à la fois irremplaçable et infiniment vulnérable. L'humanité guerrière est à son apogée lorsqu'elle produit l'unique, et elle n'espère qu'en lui. Elle s'effondre, faute de pouvoirle soustraire à la loi du commun.

\section{University of Alberta}

\section{Notes}

1 Jacques de la Taille, Alexandre, éd. C.N. Smith (Exeter: Univ. of Exeter Printing Unit, 1975). Pour Hercules Oetaeus, nous utiliserons l'édition de Léon Herrmann, Sénèque - Tragédies (1925; 5ème tirage, Paris: Les belles lettres, 1971), Tome II.

2 Antoine Hardy, “Alexandre”, dans Théâtre d'Antoine Hardy, éd. E. Stengel (Marburg, 1884), Tome IV. Jean Prévost, "Hercule", dans Les tragédies et autres oeuvres poétiques (Poitiers: Iulian Thoréar, 1613). Pierre Mainfray, Tragédie des forces incomparables et amours du grand Hercule (Troyes: Yves Girardon, 1616). Jean Rotrou, "Hercule mourant", dans Oeuvres de Jean Rotrou, éd. Viollet le Duc (Paris, 1820), Tome II.

3 Antoine Hardy, "La Mort d'Achille", dans Théâtre d'Antoine Hardy, éd. Stengel, Tome II. Isaac de Benserade, La Mort d'Achille et la dispute de ses armes (Paris: Sommaville, 1637). Antoine de Montchrestien, "Hector", dans Antoine de Montchrestien - Two Tragedies, éd. C.N. Smith (Londres: Athlone Press, 1972).

4 Morel, dans Les tragédies de Sénèque et le théâtre de la Renaissance, éd. Jean Jacquot (Paris: C.N.R.S., 1964), pp. 95-111. A l'autre bout de la chaîne, l'éditeur moderne de Jacques de la Taille ne fait aucune mention d'Hercules Oetaeus lorsqu'il examine les sources d'Alexandre (Smith, pp. XI et suiv.). Jacques de la Taille signalait pourtant, dans sa pièce même, par l'entremise de son héros, sa dette envers l'auteur latin:

Et puis je ne me dois contrister d'avantage

Si par poison je meurs, car telle fut la mort

D'Hercule mon ayeul le magnanime et fort...

\section{(v. 1126-1128)}

Souligner ainsi le parallélisme des sujets, ce n'était pas seulement indiquer leur filiation, mais aussi faire ressortir la figure tragique qui leur était commune.

5 Thomas Corneille écrira lui aussi une Mort d'Achille (voir Oeuvres de Thomas Corneille, Paris 1758; réimp. Genève: Slatkine Reprints, 1970), mais qui n'aura rien en commun, pour ce qui est de la signification tragique, avec les pièces écrites sur le même sujet au début du siècle.

6 "Cycle d'Alexandre," dans Réalisme et dramaturgie - Etude de quatre écrivains: Garnier, Hardy, Rotrou, Corneille (Paris: A.G. Nizet, 1974), pp. 104-124. La notion même de cycle, telle qu'on l'utilise communément, sacrifie à qu'il y a de plus trompeur dans le critère du sujet.

7 Jacques de la Taille s'inspire, comme le feront aussi Hardy et les auteurs des Hercule français, du texte de Sénèque, dans ce cas, des vers 1249-1262. Entre le modèle et les imitations, les ressemblances textuelles sont trop évidentes pour qu'il nous faille les signaler à chaque pas, et quant aux écarts, ils ne relèvent pas toujours du domaine de notre analyse. A moins que les variantes n'éclairent différemment le schéma commun, nous nous contenteront donc d'illustrer nos remarques d'exemples tirés d'une pièce ou d'une autre, sans aucune préférence méthodologique et sans procéder à des comparaisons.

Perdidi mortem, hei mihi,

totiens honestam: titulus extremus quis est?

(Sénèque, v. 1205-1206)

Perdi-ie tant de fois une mort honorable

Pour perdre mon beau tiltre et mourir miserable,

Tiltre de mon tombeau dont ie fus si soigneux,

Las! serez vous sali d'un trespas vergoigneux. 


\section{0 / Renaissance and Reformation}

9 La scène entre Hercule et Alcmène explicitait, chez Sénèque, le symbolisme de la tunique teinte du sang de Nessus, autant que faire se pouvait sans enlever à celle-ci son efficacité dramatique. Au moment où le héros rencontrait sa mère, l'origine magique du mal qui le dévorait s'était déjà imposée comme une réalité dans la fiction, de sorte que le doute ne semblait plus permis à son sujet sans risque d'incohérence. Alcmène se montrait neanmoins sceptique:

HER. Aditum uenenis palla femineis dedit.

Alc. Vbinam ista palla est? Membra nudata intuor.

Elle allait même jusqu'à opposer à l'explication du mal par le sortilège une explication par des causes naturelles:

Non uirus artus, nate, feminem coquit,

sed dura series operis et lungus tibi

pauit cruentos forsitan morbos labor. (v. 1396-1398)

Prévost reproduira cette scène (acte IV, p. 30) assez fidèlement pour sauvegarder l'échappée qu'elle ménageait dans l'épaisseur du sens. Plus soucieux, peut-être, de cohérence immédiate, et moins intéressé, de toute façon, par la tragédie de l'assassinat en tant que telle, Rotrou s'écartera, à cet endroit, du modèle sénéquien. Dans Hercule mourant (acte IV, sc. 2), Alcmène n'aura plus aucune peine à croire au maléfice que dénonce son fils, et la scène en question perdra, du coup, sa fonction herméneutique.

10 Dans la plupart des tragédies que nous étudions, le repos du guerrier, orginellement conçu comme l'arrêt de l'activité conquérante par épuisement de son objet, figure parmi les circonstances postulées par l'exposition. Rattaché assez vaguement à l'assassinat, à en juger seulement par l'économie apparente des intrigues, il en est une condition nécessaire et suffisante, selon le raisonnement symbolique qui sous-tend ces intrigues. Le guerrier tombe dans le piège de la mort naturelle en cessant, pour une raison ou pour une autre, d'affronter la mort visible et loyale qu'incarne l'adversaire. Comme renseigné par l'expérience des héros qui l'ont précédé dans l'histoire du théâtre tragique, l'Achille de Benserade commente lui-même les dangers auxquels l'expose l'oisiveté:

La mort dans le peril ne m'espouuante pas,

Ie la crains dans la paix, et la cherche aux combas.

Qu'elle ne vienne à moy que par la noble voye,

Ie ne la craindray pas pourveu que ie la voye,

Ie l'ay veuë effroyable, et la verrois encore,

Sans pallir ie l'ay veuë au front du grand Hector;

Mais la fine qu'elle est fait son coup dans le calme,

Souvent elle se cache à l'ombre d'une palme,

Et c'est là le suiet de ma timidité,

Ie me fie au danger, et crains la seureté. (acte I, sc. 1)

Il oubliera sa propre analyse, trois actes plus tard, et celle-ci ne ressortira que mieux, à la faveur du rappel dramatique ainsi ménagé:

Achille: Mais ie n'ay pas suiet de craindre en ce lieu-cy,

Ie ne me vis iamais plus seurement qu'icy,

Une tréue sacree est ma iuste deffence,

Et par elle s'endort la haine et la vengeance,

Ie gouste le repos des plus lasches humains,

Loin des coups, dans un Temple.

Alcimédon:

Et c'est ce que ie crains.

(acte IV, sc. 4)

11 Hercules furens, v. 1194; Hercules Oetaeus, v. 1399.

12 Déception renouvelée, dans la tradition humaniste, à l'occasion de toute enquête d'envergure sur les voies du salut. Il est alors indispensable, à titre de prolégomènes, et comme en cédant, par méthode, à une fascination millénaire, d'accorder encore une fois sa chance à l'effort sans mémoire du guerrier. Tête d'Or, le héros du jeune Claudel, est lui aussi, par exemple, de la race des invincibles, et son discours d'agonisant ("Tête d'Or," $2^{\mathrm{e}}$ version, dans Théâtre, éd. Jacques Madaule et Jacques Petit, Paris: Pléiade, 1967, Tome I, p. 279) fait écho aux discours des Hercules et des Alexandres: 
Je ne puis plus, je ne puis plus faire!

O membres rudes, maintenant brisés! Moi, moi,

Me voici à votre merci, plus faible qu'un débauché,

Qu'une ignoble motte de chandelle dont l'oeil liquoreux

verse sa flamme! Ce vil corps, cette sale machine,

Refuse à mon âme son langage!

Quelle force me manque! Tu me laisse, Vertu Royale!

13 v. 1488-1496. Hercule lègue aussi ses armes à Philoctète (v. 1648-1659), ce qui a bien l'air d'une transmission de la tâche héroïque, mais rien dans le texte même n'encourage une telle interprétation.

14 Aucune tradition prestigieuse n'obligeait les auteurs qui traitaient l'histoire d'Alexandre à finir par l'apothéose du héros. C'est là probablement l'une des raisons pour lesquelles cette histoire est entrée en concurrence, en tant que sujet de tragédie, avec l'histoire d'Hercule. Quant aux adaptateurs mêmes d'Hercules Oetaeus, ils ont dû ménager, chacun à sa manière, des compromis entre leur fidélité envers le modèle littéraire et leurs convictions religieuses. Prévost, par exemple, suit de près le dénouement que lui impose Sénèque, mais en atténue l'impact mystique, car il y fait aussi entendre la voix de l'incrédulité. Alcmène exulte, dans sa pièce, comme elle le faisait chez Sénèque:

Il est vray, c'est un Dieu, mon Hercule est un Dieu

Digne qu'on le reuere et l'adore en tout lieu.

mais Philoctète commente:

Le chetif croit tousiours ce que plus il desire.

$$
\text { (acte V, p. 50) }
$$

Chez Rotrou, l'apothéose du héros semble, au contraire, affirmée avec plus de force que chez Sénèque même: non seulement on entend Hercule parler d'outre-tombe, mais on le voit aussi descendre en personne du haut du ciel. Cette surenchère n'affiche, en fait, la lettre du dénouement sénéquien que pour mieux en estomper l'esprit. Elle est une invite à la distanciation, elle remplace le saisissement mystique par la convention mythologique. Dans Hercule mourant, le surnaturel païen est, comme dans les pièces à machines, ornemental et allégorique.

Les auteurs français n'auront aucune difficulté, par contre, à reprendre, dans leurs pièces, le mouvement d'élévation morale qui précédait, chez Sénèque, l'apothéose proprement dite du héros. Les invincibles finissent tous par affirmer leur supériorité sur l'ordre, qui les écrase perfidement, de la nature, et par récupérer ainsi sur le plan général de l'héroïsme, la dignité perdue sur le plan particulier de l'héroïsme guerrier. On aurait tort d'insister sur ce sommet de l'édifice tragique au détriment des assises. Pour apaisante et exemplaire qu'elle soit, la résignation stoïcienne ne parvient qu'à sublimer, tout au plus, le problème que posent les tragédies de l'assassinat, elle ne le résout pas.

15 L'examen de la querelle entre Don Diègue et Don Gomez à la lumière de la tradition où elle s'inscrit de toute évidence corrobore, pour l'essentiel, les conclusions que Serge Doubrovsky tirait du seul texte cornélien (Corneille et la dialectique du héros, Paris: Gallimard, 1963, pp. 90-92), mais nous empêche aussi de les accepter sans quelques réserves quant aux détails. Le problème de la "dégradation temporelle" figure, sans doute, au début du Cid comme au centre de toutes les tragédies de l'assassinat. Il ne nous semble pourtant pas tenir, ni dans l'un ni dans les autres, à la nécessité de renouveler l'épreuve héroïque - la logique du salut guerrier se fonde justement sur cette nécéssité - mais à l'inachèvement de la série d'épreuves renouvelées, soit que celle-ci épuise toutes les épreuves possibles sans rencontrer d'épreuve suprême, comme dans le cas mythique des invincibles, soit qu'elle s'arrête prématurément, avant une telle épreuve, comme dans le cas "réaliste" de Don Diègue.

16 v. 1793. 